\title{
Study on Machine Learning for Identification of Farmer's Query in Kannada Language
}

\author{
Anusha Pai \\ Department of CSE \\ NMAMIT, Nitte
}

\author{
Sarika Hegde \\ Department of CSE \\ NMAMIT, Nitte
}

\begin{abstract}
Agriculture is considered as the economic backbone of almost all the countries in the world. As there is increase in population, the need for basic requirements such as food, clothing, shelters, and medicines also increases. To meet all these needs, advancement in agriculture is needed. Various technologies have been introduced in agriculture to increase the yield of several crops. One among them is the machine learning technology. Machine learning is an important area in computer science that allows the computers to write programs on its own without being explicitly programmed. Machine learning techniques in agriculture help to improve the traditional farming techniques in a very efficient way. The main purpose of this paper is to learn different use of machine learning techniques in agriculture and to develop machine learning model for agriculture.
\end{abstract}

\section{Keywords}

Agriculture, Machine Learning, Supervised Learning, Unsupervised Learning, Speech Recognition, MFCC

\section{INTRODUCTION}

Agriculture plays a very important role in the economy of all countries in the world. It also aids to meet the basic needs of human beings by providing food, clothing, shelters, and medicines. The main aim of agriculture is to increase the yield of all the crops and to protect it from deterioration and misuse. Some of the advantages of agriculture are 1. It provides basic food requirements for the increasing population of the world. 2. It provides raw materials to the industries such as cotton for textile industry, timber for wood industries and so on. 3. It helps in foreign exchange of the country. 4. It provides job opportunities to all kinds of people and so on. Because of the increased growth in population, the demand for food has also increased. As a result new technologies are introduced to increase the productivity of agriculture

Machine learning is an area in computer science that allows computers to write programs on its own without being programmed explicitly. The main goal of machine learning techniques is to build algorithm that receives input and uses some analysis to predict the output within a range. It mainly makes decisions by detecting patterns from the past data and generalizing it in the future data. Machine learning is mainly classified in to supervised learning, unsupervised learning and reinforcement learning.

\subsection{Supervised Learning}

This is a type of machine learning technique where we will have input variable $\mathrm{x}$ and output variable $\mathrm{y}$ and this algorithm generate a function that will predict the results based on the input values. Supervised learning is mainly classified in to regression and classification. A supervised learning problem is said to be a regression problem, if the output value is a continuous value such as weight, height and so on. Similarly a supervised problem is said to be a classification problem if the output value is a discrete values such as male or female, disease or non-disease and so on. Some of the classification algorithms include support vector machines (SVM), neural networks, naive bayes classifier, decision trees, $\mathrm{K}$ nearest neighbors and so on. Some of the regression algorithms include linear regression, nonlinear regression, decision trees, and neural networks.

\subsection{Unsupervised Learning}

This is a type of machine learning algorithm where, we draw implications from the input data without containing labeled responses. Unsupervised learning algorithms are classified in to cluster analysis and association. A cluster analysis is a one where objects which are similar to each other are grouped together. Association rule is the one where the existence of interesting relationships between the variables in the dataset is discovered. Some of the examples of unsupervised learning algorithms are hierarchical clustering, K-means clustering, gaussian mixture models, self-organizing maps, hidden markov models, apriori algorithm and so on.

\subsection{Reinforcement Learning}

It is a type of machine learning algorithm that allows the machine to learn its behaviour from the feedback that is obtained from the environment. Some of the algorithms that come under Reinforcement learning are Q-learning, Temporal difference, Deep Adversarial Networks and so on.

Speech recognition is one of the major applications of machine learning. Speech recognition is the ability of the machine or program to recognize the spoken words or phrases and convert them into machine readable format. Using speech recognition in agriculture helps farmers in many ways. Some of its advantages are, it helps farmers to gain access to relevant information in local languages, and it helps agribusiness people to obtain feedback from farmers for their products and so on.

In this paper we discuss how various machine learning techniques are useful in agriculture and develop speech recognition model to agricultural data. The remaining section of the paper is organized as follows. In section 2, we discuss the work done by the researchers. In section 3, we discuss how speech recognition can be applied to agricultural dataset in Kannada language using machine learning technique and how features can be extracted using MFCC. In section 4, we discuss the results obtained after extracting the features using MFCC and we conclude in section 5. 


\section{LITERATURE SURVEY}

Much research has been conducted in the field of agricultural domain using various machine learning techniques by several researchers. In this section, we focus on the previous work done by several researchers in this field.

\subsection{Disease detection in crops using machine learning}

Machine learning techniques have been used in the detection of diseases crops from the past several years and their applications have proved to be worthwhile. Henmi, Tomohiro, et al [1] have explained how SVM is used in the early detection of plant faults. Fault detection in this research involves classification between the normal state and fault state. In the early stage of learning, only normal state data are available, and the fault state data are gained a little seconds later after the error occurs. For classification of these kinds of cases where only single side of data is available, one class SVM is used. To get high ability, a new kernel function called generalized Gaussian function is used. Huang, Tisen, et al [2] and Islam, Monzurul, et al [3] have explained how SVM is used in the early detection of plant faults such as detecting sugarcane borer diseases in sugarcane crops and detecting diseases in potato plants. These approaches involves taking the images of seeds of plant leaf as the input and perform segmentation on those images and with the help of SVM, different kind of diseases are detected and distinguished. Wang, Xiang, et al [4] have presented a study on how SVM technique is used in controlling diseases in maize crop. Southern corn beam is the disease that is usually found in maize crops and to solve this problem several combinations of fungicides are used. This approach involves constructing a model called bipolar maydis using SVM for choosing the optimal combination of fungicides.

Rodríguez, Jhonn Pablo, et al [5] and Lasso, Emmanuel, and Juan Carlos Corrales [6] have explained how machine learning techniques are used in increasing the coffee rust samples and detection of coffee diseases. One of the problems faced by the coffee farmers is the lack of coffee samples required for detection of coffee plant diseases. To solve this problem, supervised learning algorithms are used which provides a guideline to increases the coffee rust samples and then semi supervised learning technique is used in the detection of coffee diseases. This approach involves constructing a set of rules to identify pests and diseases from coffee, which are represented in terms of graphs and later to determine the probability of diseases and pest in coffee plants similarity techniques using graphs, are used.

Patil, Suyash S, and Sandeep A. Patil, Suyash S., and Sandeep A [7] Thoratunsupervised learning algorithm is used in early detection of grape diseases. The unsupervised algorithm used in this approach is the hidden markov model. This approach involves building a monitoring system which uses hidden markov model in early detection of grape diseases and provides information to the farmers through SMS.

\subsection{Automatic Irrigation using machine learning}

We require huge amount of water for irrigation in agriculture, but water levels of earth are decreasing day by day due to global warming and so on. To solve this problem machine learning techniques are used. Goldstein, Anat, et al [8] have explained how Machine learning techniques can be effectively used in irrigation. In this paper, data is collected through all the sensors present in the field and later dataset is created by integrating these data. The dataset created consists of several information regarding sensor, weather, plots, irrigation and so on. Later irrigation prediction models were developed using traditional linear regression, GBRT, BTC. Navarro-Hellín, Honorio, et al [9] have presented a paper on how a decision support system is used in irrigation management. This paper involves developing a decision support system for irrigation managment in agriculture, which helps in easy estimation of the weekly irrigation needs, based on soil measurements and various climatic parameters. These parameters are obtained through different nodes deployed in the field. Closed loop control scheme is used by this system for adapting the decision support system in estimating errors. Later PLSR and ANFIS techniques are used in this approach.

\subsection{Automatic crop yield prediction}

Selection of appropriate crops plays an important role in increasing the yield of crops. It depends on various factors such as quality of soil, temperature, geography of the region, climatic conditions and so. Machine learning algorithms play an important role in crop yield prediction. Kumar, Rakesh, et al [10] have explained how Machine learning techniques are used in the selection of crops which in turn increases the yield rate. In this paper, it helps the farmers to solve the crop selection problem by using Crop Selection Method (CSM) based on various machine learning algorithms. The inputs taken in this research are crops, sowing time, the predicted yield rate and plantation days which results in a sequence of crops which increases the net yield of the crops per year. Shakoor, Md Tahmid, et al [11] have explained how supervised machine learning techniques is used in predicting the output of agricultural products. This research helps the farmers of Bangladesh in deciding which crop is suitable for a particular area based on previous year's data which is taken from Bangladesh agriculture council. The main purpose of system is to help farmers in increasing their profit by selecting maximum yield giving crops. The input taken in this research is a dataset which includes details of crops yield per hectare, rainfall, temperature, and region and so on. These data are analysed using supervised machine learning techniques. After analyzing, the algorithm gives the result, which predicts the profitable output.

Pantazi, Xanthoula Eirini, et al [12] an. Romero, José R., et al [13] have explained how machine learning techniques are used in guessing the yield of wheat crops. X.E. Pantazi, D. Moshou have explained about the sensing techniques used in predicting the yield of wheat crops. Different crop and soil sensors are placed in the field to obtain high resolution data about soil and crops. Self-Organizing Map models used in this approach are counter propagation artificial networks, $\mathrm{XY}$ fusion, supervised kohonen networks and so on. Supervised learning methods are used to combine data about crops and soil with the isofrequency classes of wheat. An online infrared spectroscopy sensor is used in the implementation of this approach. Whereas Romero, Roncallo have have explained how Apriori algorithm is used in predicting yield of durum wheat in the province of Buenos Aires. The main goal of this research is to use several machine learning algorithms in predicting durum wheat yield by obtaining several rules. The data collected by the research group were run on apriori algorithm and the results indicate that apriori algorithm obtains best results for all locations and the classifiers used by different algorithms.

Veenadhari, S., Bharat Misra, and C. D. Singh [14] have focussed on how Machine learning techniques are used for forecasting the harvest of the crops based on the climatic 
parameters. In this paper, it helps the farmers of India to predict the influence of various climatic conditions on yield of various crops. This research involves the development of a software tool called 'Crop Advisor', which is a user friendly webpage for predicting the influence of climatic conditions on crop yields using C4.5 algorithm. This research is mainly conducted in the Madhya Pradesh Region of India. The inputs taken in this research are data which contains information regarding the yield of particular crop from last 20 years for that particular region and the climatic parameters such as rainfall, maximum and minimum temperature and so on for that particular region from Indian Meteorological Department.

\subsection{Increasing the productivity of agriculture using machine learning}

Machine learning techniques are used in increasing the productivity of agriculture in almost all countries of the world. Sirsat, M. S., et al [15] have explained how supervised learning algorithms such as SVM, decision trees, nearest neighbor classifiers and so on helps the farmers in making decisions regarding improving soil quality and crop quality by classifying the soil parameters. The input taken in this approach are the various soil parameters such as village wise fertility indices of organic carbon (OC), phosphorus pent oxide (P2O5), manganese (Mn) and iron (Fe), soil $\mathrm{pH}$ and type, soil nitrous oxide $(\mathrm{N} 2 \mathrm{O})$ and potassium oxide $(\mathrm{K} 2 \mathrm{O})$.

Lanorte, Antonio, et al [16] have explained how the productivity of agriculture can be increased by using SVM technique in the estimation of agricultural plastic waste with the help of landstat 8 images. The usage of plastics in agriculture has many benefits, as it is used in protection of crops, mulching of soil, used in pipes which are used for irrigation, silage covering, harvesting and so on. Since the usage of plastics is done in large quantities, the outcome is the huge amount of agricultural plastic waste which needs to be disposed. But the main problem here is lack of presence of input and output data on the use of plastics in agriculture and the poor management schemes used. To solve this problem, this research aims in estimating and mapping agricultural plastic waste using satellite images. Using this method we can easily obtain the updated waste maps, which helps the waste managers to use this information create a waste management plan which includes defining the position of collections centers and waste route maps. Support vector machine is used for image classification to detect the various plastic covering materials used.

Purandare, Himanshu, et al[17] have explained how Machine learning system is used in the analysis of post-harvest losses. Iot and machine learning techniques are used in this approach. This paper involves building an end to end system, which consist of a system that notifies regarding crops. It also consists of a prediction system which includes details regarding the stocks which are to be dispatched correctly, temperature and humidity required at which crops can be transported and so on. Classification and regression techniques are used for prediction analysis.

Yahata, So, et al [18] has explained how a hybrid machine learning approach can be used for automatic plant phenotyping in smart agriculture. This research has considered 2 image sensing methods, one is for flower detection and the other one is for seedpod detection. In this paper, a mixture approach using different image processing and machine learning techniques are used in detecting plant phenotyping. The flower detection method involves developing a hybrid system consisting of SLIC and CNN methods, whereas seedpod detection involves developing a hybrid system consisting of SLIC and Viola-Jones object discovery technique.

\subsection{Classification of agricultural parameters using machine learning}

Machine learning techniques used in classification in agriculture helps in improving the productivity of the crops. Arango, R. B., et al [19] have explained how the land zones are classified in to either arable or non-arable land using supervised learning techniques. This approach involves taking landsat 8 satellite images as inputs, creating datasets from these images and later applying SVM, neural networks for classification of land zone in to arable or non-arable land. Qader, Sarchil H., et al [20] have explained how SVM technique is used in classifying vegetation type in Iraq with the help of satellite-based phonological parameters. Iraq is an example of arid-semiarid region, where there is no specific system for predicting the cropland distribution. To solve this problem, this research aims at developing phenology based classification using SVM for cropland spreading in Iraq.

Machine learning techniques are used in the classification of weeds and crops. De Rainville, François-Michel, et al [21] has explained how unsupervised learning algorithm is used in discriminating between weeds and crops in the field. This approach involves using data from rows and inters rows of the farm and applying Naive bayesian classifier to these data to discriminate between crops and weeds and at last unsupervised learning technique is used to refine the preceding classification. Athani, Suhas S., and Ch Tejeshwar [22] have explained how SVM technique is used in discriminating between weeds and maize crops. Here the inputs taken are the datasets consisting of images of maize crops and weeds. In order to separate maize crops from weeds, several features such as shape, texture and colour are used. Several statistical texture features such as intensity, mean, energy, entropy standard deviation and so on are used in this approach. Later SVM classifier is used in predicting whether the plant is a maize crop or a weed. Similarly Cheng, Beibei, and Eric T. Matson [23] have explained how Clustering algorithm is used in the automatic discrimination of rice and weed crops. This research explains how a machine learning technique is used to discriminate between rice and weeds. In this, at first Harris Corner Detection algorithm is used to find the point of intersects, then some of the features are extracted which is then fed in to the machine learning algorithm to differentiate between weed and rice crops and at the end, a clustering algorithm is used for noise removal.

Conţiu, Ştefan, and Adrian Groza [24] have explained how classification of crops with remote sensing can be improved with the help ensemble learning. This research involves development of hybrid system that uses expert knowledge regarding agricultural and machine learning algorithms. Vote based methods are usually used to solve conflicts between ensemble's learners, but this method is not applicable in all cases. To solve these kinds of problems we use ensemble learning with argumentation based conflict resolution techniques. The machine learning techniques used here are neural networks, support vector machines and decision tree.

Shastry, K. Aditya, H. A. Sanjay, and G. Deexith [25] have explained how a SVM based on kernel is used in classifying datasets of agriculture with continuous attributes. The input taken in this research is the dataset which contains information about the soil, seeds and land cover. At first, preprocessing of the agricultural dataset is done to remove all 
the redundant values and later gradient descent methods are used to find the SVM parameters and C. The kernel method proposed in this research is the quadratic radial basis kernel which consists of both the quadratic and radial basis functions.

\subsection{Speech recognition in agriculture}

Applications of speech recognition in local languages have helped farmers in many ways. Farmers can easily get answers to most of their question in local languages without having much educational qualifications. Mohan, Aanchan, et al [26] have explained about the acoustic modeling of speech recognition in Indian languages for farming sector. The multiple languages used here are Hindi and Marathi. These two languages are used because they are linguistically related languages. At first speech data regarding agricultural commodities task domain is collected, and later cross corpus acoustic normalization method is applied. This results in a system which delivers the best performance for both the languages.

Shrishrimal, Pukhraj P., Ratnadeep R. Deshmukh, and Vishal B. Waghmare [27] have explained about building speech database for agricultural purpose using Marathi language. The various procedures followed in this approach are 1. Development of text corpus 2. Speech data collection 3 . Recording the speech data and 4. Signal enhancement. Development of text corpus is done by collecting all the Marathi words related to agriculture by visiting various websites regarding agriculture. The words selected from the websites were classified into different groups. Later the farmers of Aurangabad district of Marathwada region of Maharashtra state were selected as the speakers and they were told to to speak the selected words with 3 utterance of each word. The speakers included both females and males. Then the words from different speakers were recorded. And then the recorded speech was enhanced to remove the noise present. G Gaikwad, Santosh, Bharti Gawali, and Suresh Mehrotra [28] have explained about building a speech recognition system for agriculture sector in Marathi language. Here, at first speech data in Marathi language is created. The speech database created consists of 3 sets of databases, one for names of the crops, next for queries regarding toxicities about the crops and third for name of the disease and its solutions. Totally 35 speakers were selected out of which 18 were males and 17 were females. Later the agro based interactive system is built based on the above information, which helps the farmers in obtaining the solutions to their problems in their mobile phones very easily.

Imran, Ahmed, and Sunil Kumar Kopparapu [29] have explained how natural language speech interface is built to get market information. The proposed system is named as mandi bhav jhankari system. This research helps the farmers in getting the information regarding the prices of various commodities in hindi language easily. The inputs taken in this approach are commodity name and name of the mandi. With the help of these two inputs we can easily obtain the information regarding the price of commodities at a particular region using mandi price database which needs to be regularly updated. In this system, at first the farmer asks query in a natural way. Later ASR engine is used to recognise the spoken query. Then the NLU is used to extract price of the commodity and mandi name from the inputted query. Later, these 2 parameters are passed to the fetch price block which is used to fetch the price of a particular commodity in a particular place. At last the output is processed and sent to the farmer in Hindi language.
Godambe, Tejas, and K. Samudravijaya [30] have explained about how a voice based agricultural information system is built by collecting speech data from the Maharashtra farmers. The voice based agricultural information retrieval system consists of 3 sub systems automatic speech recognition engine to identify the mandi, district and name of the commodity, a web interface to get commodity prices from the market website and a Text to speech system to display the commodity price to the farmer. The data is collected from the farmers by asking them different kinds of questions such as direct questions, picture prompted questions, prompted questions, general questions and so on.

Mantena, Gautam Varma, et al [31] have explained about how a conversation system based on speech is developed for accessing commodity prices in agriculture. This research involves developing a speech based conversation system which takes speech in Indian languages as an input, through which farmers can easily interact with the system. When a user asks query, the inputted speech is converted in to a text by automatic speech recognition unit and the necessary information is extracted from this by parsing using NLU unit. Later, the necessary actions and response to be given to the user is determined by the DM unit. The NLG unit is used to give responses to the user in the form of sentences. Later the generated sentences are synthesized by TTS module. Mandal, Partho, et al [32] have explained about how deep neural networks can be used for developing speech recognition system for agricultural commodities in Hindi language. Here at first speech data regarding price of agricultural commodities is collected in Hindi language. Later acoustic modeling is built with the combination of deep neural network and deep neural networks. After that 3 stage training process is performed which consists of frame cross-entropy training, RBM pre-training and sequence-training optimizing MMI/sMBR. After repeating the experiment for several times, the accurateness of the system has come out to be $82 \%$.

$\mathrm{Xu}$, Jinpu, et al [33] have explained how speech recognition can be applied to agricultural data. This research involves applying speech recognition technology for the information collected over agricultural prices for several agricultural products. In this at first, a speech corpus is constructed, then a decoder unit is used to train hidden markov to differentiate between male and female voices. Later to solve some of the problems caused by insufficiencies of training models decision tree is used. Later CMN and CVN methods were used in reducing the mismatch between testing and training unit. Using speech recognition for agricultural data includes many advantages such as it reduces the staff, improves the work efficiency, easy to operate and so on.

Yadava, Thimmaraja G., and H. S. Jayanna [34] have explained about building a spoken query system for agriculture in Kannada language. Automatic speech recognition models are built by means of kaldi speech recognition toolkit. The input in the form of speech for this approach is collected from different Karnataka regions. The information regarding commodity prices and weather are collected from AGMARKNET and IMD websites. Acoustic models created using kaldi involves speech transcription, speech Validation, dictionary and phoneme set creation in Kannada language , extracting features using MFCC, Testing and training using kaldi and so on.

Ou, Wenhao, et al [35] have explained about the implementation of agricultural information system based on speech recognition using Microsoft speech development platform. Here the Microsoft SAPI speech recognition system 
is used. We need command mode to get accuracy in the speech recognition and in the design process. Keywords are generated using $\mathrm{xml}$ format. This system provides an easy way for the agricultural workers to access timely information regarding agriculture in a very efficient way.

Based on the literature survey we found that Machine learning is an interesting field that can be used to solve many real time problems. Applying machine learning techniques in agriculture has solved most of the agricultural problems and it has proved to be a huge advantage for farmers. Some of the advantages of using machine learning techniques in agriculture are, it helps in the early detection of diseases in crop which avoids huge amount of loss to farmers, helps in increasing the yield of crops, helps in solving irrigation problems, using speech recognition helps farmers to ask queries regarding agriculture in local languages and so on.

\section{METHODOLOGY}

\subsection{Problem Statement}

In our work, we are interested in developing machine learning model which can be used to automatically respond to the farmer's query. The query can be asked in local languages, that is Kannada language. The main keywords used in this approach are crop names and district names of Karnataka state. The queries which can be asked in this approach are regarding price of commodities for important crops in some districts of Karnataka region.

The proposed methodology involves building a speech recognition system for agricultural dataset in Kannada language. The data set created consists of the name of the crops and name of the districts of Karnataka state. The steps followed in building the speech recognition model are,

3.1.1Creating dataset in Kannada language.

3.1.2Feature extraction using MFCC.

3.1.3Machine learning algorithm.

3.1.4Output.

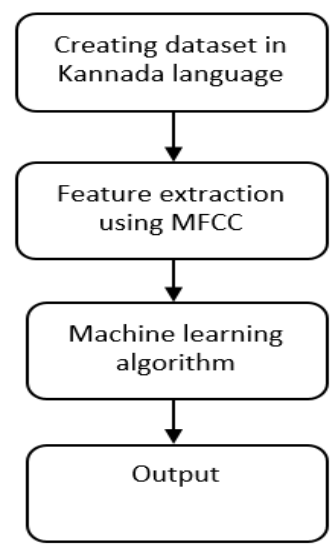

Fig 1: Speech recognition Process

\subsubsection{Dataset creation}

The dataset is created in Kannada language. The dataset consists of 10 crop names and 10 district names of Karnataka region. Recordings of each of these words were done by selecting 5 speakers. Each speaker was asked to speak 20 words with 1 utterances of each word. Therefore the total number of words recorded was 100 , where each speaker recorded 20 words.
Table 1: List of Crop names and District names

\begin{tabular}{|c|c|}
\hline \multicolumn{2}{|c|}{ Words present in dataset } \\
\hline Crop names & District names \\
\hline Paddy & Dakshina kannada \\
\hline Areca nut & Udupi \\
\hline Groundnut & Dharwar \\
\hline Maize & Shimoga \\
\hline Ragi & Mysore \\
\hline Jowar & Bijapur \\
\hline Sugarcane & Mandya \\
\hline Cotton & Chitradurga \\
\hline Sunflower & Hassan \\
\hline Wheat & Belgaum \\
\hline
\end{tabular}

\subsubsection{Feature extraction}

It is considered as the main part of speech recognition systems. The main work of feature extraction is to extract those features from the speech which helps in identifying the speakers. Each person has different characteristics embedded in utterances; as a result speech from each person varies from other person. To reduce these variations feature extractions are performed. The feature extraction used in this approach is Mel Frequency Cepstrum Coefficients (MFCC). MFCC is the most prominent feature extraction method used in speech recognition [36]. It takes speech samples as inputs, processes them and calculates the co-efficient unique to each samples. The working of MFCC is as follows.

1. Pre-emphasis: Here, the speech samples are delivered over a filter which emphasis greater frequencies. It will rise the energy of signal at greater frequency.

2. Frame blocking: In this step, the speech signal is distributed in to multiple frames. The duration of each frame will be around $20-30 \mathrm{~ms}$. The speech signal is separated into $\mathrm{N}$ samples and the neighboring frames are divided by $\mathrm{M}$. Typical values of $\mathrm{M}=100$ and $\mathrm{N}=56$.

3. Hamming windowing: In this step, the above obtained frames are multiplied with to keep the continuousness of the start and end points of frame of the speech signal. The hamming window is given as $(n)=0.54-0.46 \cdot \cos (2 \pi n N-1), 0 \leq n \leq N-$ 1

4. Fast Fourier Transform: It is the process of transforming time domain to frequency domain. We perform this step to get the magnitude frequency reply for individual frames.

5. Triangular Band pass filters: This step is used to smooth magnitude spectrum by multiplying magnitude frequency domain by a set of 20 triangular band pass filters.

6. Discrete Cosine Transform: Here, we apply DCT to the $20 \log$ energy $E_{k}$ which is gained from the 
triangular band pass filters which gives L-mel-scale cepstral coefficients. The equation for DCT is,

$\left.\mathrm{C}(\mathrm{n})=\sum \mathrm{Ek}^{*} \cos (\mathrm{n} *(\mathrm{k}-0.5) * \pi / 20)\right)$

where $\mathrm{n}=0,1,$. to $\mathrm{N}$ where $\mathrm{N}$ is the count of triangular bandpass filters, $\mathrm{L}$ is the count of mel-scale cepstral coefficients.

In this approach, each speech sample is passed to MFCC and 20 features from each sample is extracted with frame size. Frame size depends upon the length of speech samples. Later the frames of speech sample need to be normalized and passed to machine learning algorithms.

\subsubsection{Machine learning algorithms}

Numerous machine learning algorithms are used to classify speech data. The best machine learning algorithm which can be used is Long Short-Term Memory Networks (LSTM). LSTM is a distinct kind of RNN accomplished of learning long term dependencies. LSTM's were mainly developed to solve the problem of long dependencies. It is mainly considered as the extension of RNN, which extends its memory. Like RNN, LSTM too have a chain like structure, but the reiterating module in LSTM has 4 neural networks interrelating in a very special way instead of a single neural network. The most important part of LSTM is cell state and 3 gates such as input gate, output gate and forget gate, which are used to protect and control the cell state [37]. LSTM consists of following steps:

1. The foremost step in LSTM involves deciding what data we need to dispose from the cell state. This choice is made with the help of sigmoid layer called forget layer.

2. The following step is to decide what new data is to be kept in the cell state. It consists of 3 parts. The first part consists of a sigmoid layer called input gate layer which is used to adopt which values need to be updated. The second part consists of a tanh layer which is used to create a new vector for new values. Later these 2 parts are combined to create an upgrade to the state.

3. The last step is to decide what values are to be outputted. The result will be based mainly on our cell state.

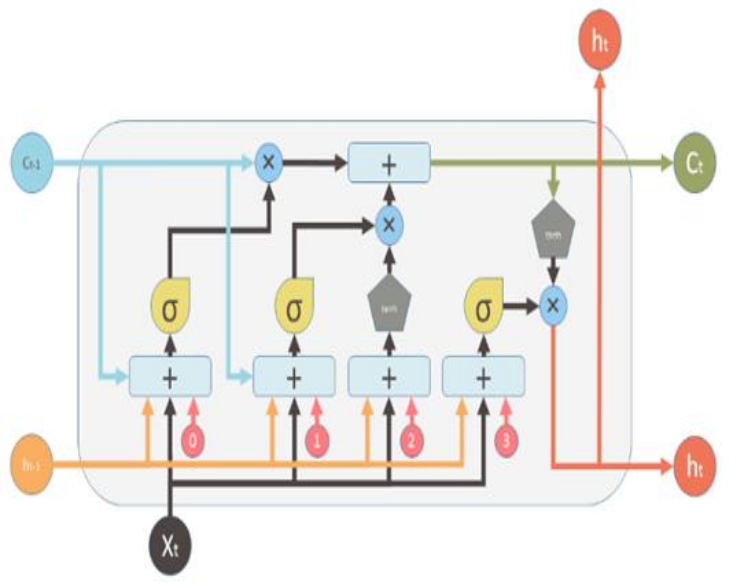

Fig 2: LSTM Building Block

\section{RESULTS AND DISCUSSION}

The results obtained after applying MFFC to above mentioned dataset is 20 unique co-efficient for each speech sample. The number of speech samples present in the dataset is 100 . The frame size of each frame is different and it depends upon the length of each speech sample.

A graph is plotted which displays the MFFC's features for the entire crop names speech data sample. In this graph, $\mathrm{x}$-axis symbolizes the number of frames and $y$-axis symbolizes the feature vectors for each frames.

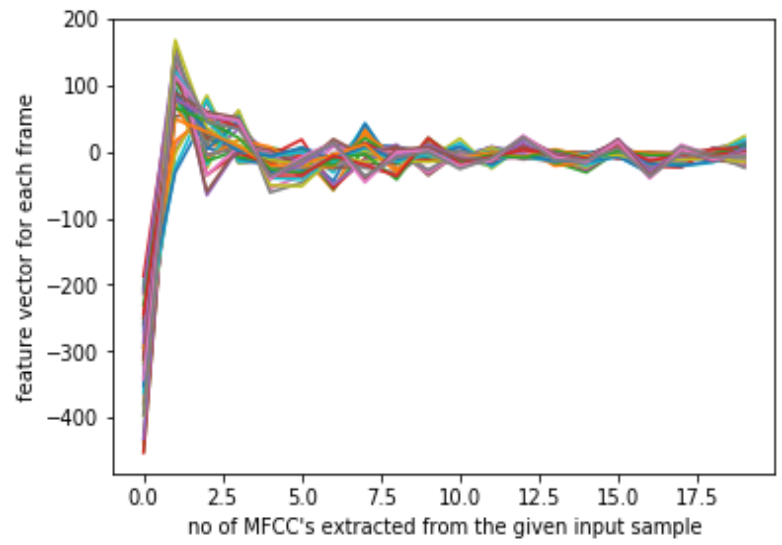

Fig 3 MFFC's obtained for crops speech data

Similarly, we have plotted a graph, which displays the MFFC's features for the entire district names speech data sample. In this graph, $\mathrm{x}$-axis symbolizes the number of frames and $y$-axis symbolizes the feature vectors for each frames.

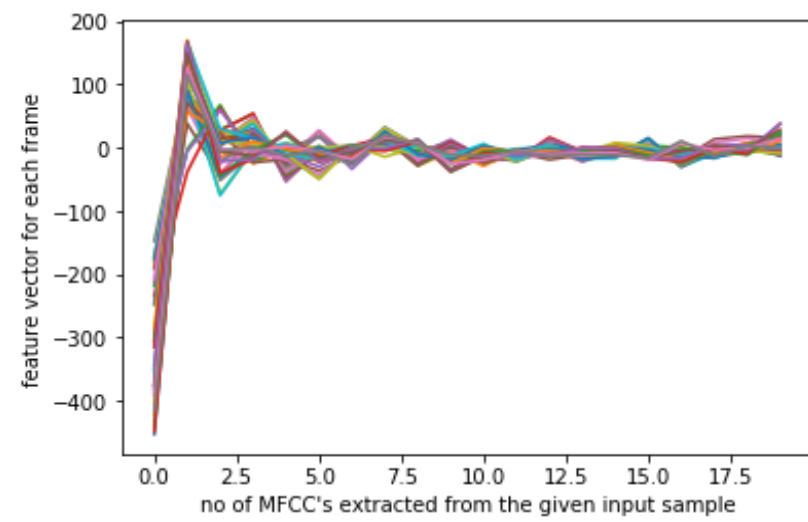

Fig 4 MFFC's obtained for district speech data

The frame size of each sample is to be normalized, that we get constant frames for all the speech samples. After normalizing we got the constant frame size as 38 . Now each speech samples consists of 20 MFCC co-efficient and 38 frames and this matrix is passed to machine learning algorithm.

\section{CONCLUSION AND FUTURE WORK}

Farming field has been missing enormous adoption of technologies and their advancements. Various technologies need to be applied to the agriculture domain in order to increase the yield of several crops. Machine learning is a technology that helps the farmers in various ways. This research has analyzed the solicitations of machine learning techniques in agricultural domain and it also gives an understanding regarding the problems faced by the farmers and how those problems are solved using several machine learning techniques. Using speech recognition in agriculture has helped farmers in many ways. It helps farmers to ask queries regarding price of the commodity, weather information in local languages and so on. The future scope of this paper is to build speech recognition system in Kannada 
language using LSTM network, so that farmers can easily ask queries regarding price of various commodities across the different districts of Karnataka state.

\section{REFERENCES}

[1] Tomohiro Henmi, Akira Inoue, Mingcong Deng "Early detection of plant faults by using machine learning." Advanced Mechatronic Systems (ICAMechS),2016 International Conference on. IEEE, 2016.

[2] Tisen Huang, Rui Yang, Wenshan Huang, Yiqi Huang, Xi Qiao "Detecting Sugarcane Borer Diseases Using Support Vector Machine." Information Processing in Agriculture (2017).

[3] Monzurul Islam, Anh Dinh, Khan Wahid, Pankaj Bhowmik "Detection of potato diseases using image segmentation and multiclass support vector machine." Electrical and Computer Engineering (CCECE), 2017 IEEE 30th Canadian Conference on. IEEE, 2017.

[4] Iang Wang, Jia Ma, Xiaowei Li, Xiaodong Zhao, Zongli Lin, Member, IEEE, Jie Chen, and Zhifeng Shao "Optimization of chemical fungicide combinations targeting the maize fungal pathogen, Bipolaris maydis: a systematic quantitative approach." IEEE Transactions on Biomedical Engineering 62.1 (2015): 80-87.

[5] Jhonn Pablo Rodríguez, Edwar Javier Girón, David Camilo Corrales, and Juan Carlos Corrales "A Guideline for Building Large Coffee Rust Samples Applying Machine Learning Methods." International Conference of ICT for Adapting Agriculture to Climate Change. Springer, Cham, 2017.

[6] Lasso, Emmanuel, and Juan Carlos Corrales. "Towards an Alert System for Coffee Diseases and Pests in a Smart Farming Approach Based on Semi-supervised Learning and Graph Similarity." International Conference of ICT for Adapting Agriculture to Climate Change. Springer, Cham, 2017.

[7] Patil, Suyash S., and Sandeep A. Thorat. "Early detection of grapes diseases using machine learning and IoT." Cognitive Computing and Information Processing (CCIP), 2016 Second International Conference on. IEEE, 2016

[8] Anat Goldstein, Lior Fink, Amit Meitin, Shiran Bohadana. "Applying machine learning on sensor data for irrigation recommendations: revealing the agronomist's tacit knowledge."Precision Agriculture: 124.

[9] H. Navarro-Hellín, J. Martínez-del-Rincon, R. DomingoMiguel, F. Soto-Valles. "A decision support system for managing irrigation in agriculture" Computers and Electronics in Agriculture 124 (2016): 121-131.

[10] Rakesh Kumar1 , M.P. Singh, Prabhat Kumar. "Crop Selection Method to maximize crop yield rate using machine learning technique." Smart Technologies and Management for Computing, Communication, Controls, Energy and Materials (ICSTM), 2015 International Conference on. IEEE, 2015.

[11] Md. Tahmid Shakoor, Karishma Rahman, Sumaiya Nasrin Rayta, Amitabha Chakrabarty "Agricultural production output prediction using Supervised Machine Learning techniques." Next Generation Computing Applications (NextComp), 2017 1st International
Conference on. IEEE, 2017.

[12] X.E. Pantazi, D. Moshou, T. Alexandridis."Wheat yield prediction using machine learning and advanced sensing techniques." Computers and Electronics in Agriculture 121 (2016): 57-65

[13] Jose R. Romero, Pablo F. Roncallo, Pavan C. Akkiraju ."Using classification algorithms for predicting durum wheat yield in the province of Buenos Aires." Computers and electronics in agriculture 96 (2013): 173-179.

[14] Veenadhari, S., Bharat Misra, and C. D. Singh. "Machine learning approach for forecasting crop yield based on climatic parameters." Computer Communication and Informatics (ICCCI), 2014 International Conference on. IEEE, 2014

[15] M.S. Sirsat ,E. Cernadas , M. Fernández-Delgado , R. Khan"Classification of agricultural soil parameters in India." Computers and Electronics in Agriculture 135 (2017): 269-279.

[16] Antonio Lanorte, Fortunato De Santis, Gabriele Nolè, Ileana Blanco"Agricultural plastic waste spatial estimation by Landsat 8 satellite images." Computers and Electronics in Agriculture 141 (2017): 35-45.

[17] Himanshu Purandare, Niranjan Ketkar, Shreyas Pansare. "Analysis of post-harvest losses: An Internet of Things and machine learning approach." Automatic Control and Dynamic Optimization Techniques(ICACDOT), International Conference on. IEEE, 2016.

[18] Yahata, Tetsu Onishi, Kanta Yamaguchi, Seiichi Ozawa. "A hybrid machine learning approach to automatic plant phenotyping for smart agriculture." Neural Networks (IJCNN), 2017 International Joint Conference on. IEEE, 2017.

[19] R. B. Arango, A. Campos, R. Canas, E. F. Combarro1"Automatic arable land detection with supervised machine learning." Earth Science Informatics 9.4 (2016): 535-545.

[20] Sarchil H. Qader, Jadunandan Dash, Peter M. Atkinson, and Victor Rodriguez-Galiano. "Classification of vegetation type in Iraq using satellite-based phenological parameters." IEEE Journal of Selected Topics in Applied Earth Observations and Remote Sensing 9.1 (2016): 414 424.

[21] Franc, ois-Michel De Rainville, Audrey Durand, Fe'lixAntoine Fortin."Bayesian classification and unsupervised learning for isolating weeds in row crops." Pattern Analysis and Applications 17.2 (2014): 401-414.

[22] Athani, Suhas S., and Ch Tejeshwar. "Support Vector Machine-Based Classification Scheme of Maize Crop." Advance Computing Conference (IACC), 2017 IEEE 7th International. IEEE, 2017.

[23] Cheng, Beibei, and Eric T. Matson. "A feature-based machine learning agent for automatic rice and weed discrimination." International Conference on Artificial Intelligence and Soft Computing. Springer, Cham, 2015.

[24] Conţiu, Ştefan, and Adrian Groza. "Improving remote sensing crop classification by argumentation-based conflict resolution in ensemble learning." Expert Systems with Applications 64 (2016): 269-286.

[25] Shastry, K. Aditya, H. A. Sanjay, and G. Deexith. 
"Quadratic-radial-basis-function-kernel for classifying multi-class agricultural datasets with continuous attributes." Applied Soft Computing 58 (2017): 65-74.

[26] Aanchan Mohan, Richard Rose, S Umesh. "Acoustic modelling for speech recognition in Indian languages in an agricultural commodities task domain." Speech Communication 56 (2014): 167-180.

[27] Shrishrimal, Pukhraj P., Ratnadeep R. Deshmukh, and Vishal B. Waghmare. "Marathi Isolated Words Speech Database for Agriculture Purpose." International Journal of Engineering Innovations and Research 3.3 (2014): 248.

[28] Gaikwad, Santosh, Bharti Gawali, and Suresh Mehrotra. "Speech Recognition for Agriculture based Interactive Voice Response System." International Journal of Modern Engineering Research (IJMER) 4.2 (2014).

[29] Imran, Ahmed, and Sunil Kumar Kopparapu. "Building a natural language hindi speech interface to access market information." Computer Vision, Pattern Recognition, Image Processing and Graphics (NCVPRIPG), 2011 Third National Conference on. IEEE, 2011.

[30] Godambe, Tejas, and K. Samudravijaya. "Speech data acquisition for voice based agricultural information retrieval." Proc. Of 39th All India DLA Conference, Punjabi University, Patiala, June. 2011.

[31] Gautam Varma Mantena, S. Rajendran, B. Rambabu . "A speech-based conversation system for accessing agriculture commodity prices in Indian languages." Hands-free Speech Communication and Microphone
Arrays (HSCMA), 2011 Joint Workshop on. IEEE, 2011.

[32] Partho Mandal, Shalini Jain, Gaurav Ojha, Anupam Shukla."Development of Hindi speech recognition system of agricultural commodities using deep neural network." Sixteenth Annual Conference of the International Speech Communication Association. 2015.

[33] Jinpu XU, Yeping, Hailong, Junfeng. "An approach of agricultural price information collection based on speech recognition." Natural Computation (ICNC), 2014 10th International Conference on. IEEE, 2014.

[34] Yadava, Thimmaraja G., and H. S. Jayanna. "A spoken query system for the agricultural commodity prices and weather information access in Kannada language." International Journal of Speech Technology 20.3 (2017): 635-644.

[35] Wenhao Ou, Wanlin Gao, Zhen Li, Shuliang Zhang, Qing Wang . "Application of keywords speech recognition in agricultural voice information system." Computational Intelligence and Natural Computing Proceedings (CINC), 2010 Second International Conference on. Vol. 2. IEEE, 2010.

[36] Wolf, Jared J. "Speech signal processing and feature extraction." Spoken Language Generation and Understanding. Springer, Dordrecht, 1980. 103-128.

[37] Graves, Alex, Abdel-rahman Mohamed, and Geoffrey Hinton. "Speech recognition with deep recurrent neural networks." Acoustics, speech and signal processing (icassp), 2013 ieee international conference on. IEEE, 2013 